\title{
Failure locus of polypropylene nonwoven fabrics under in-plane biaxial deformation
}

\author{
Alvaro Ridruejo ${ }^{\mathrm{a}}$, Carlos González ${ }^{\mathrm{a}, \mathrm{b}}$, Javier Llorca ${ }^{\mathrm{a}, \mathrm{b}, *}$
}

\begin{abstract}
A B S T R A C T
The failure locus, the characteristics of the stress-strain curve and the damage localization patterns were analyzed in a polypropylene nonwoven fabric under in-plane biaxial deformation. The analysis was carried out by means of a homogenization model developed within the context of the finite element method. It provides the constitutive response for a mesodomain of the fabric corresponding to the area associated to a finite element and takes into account the main deformation and damage mechanisms experimentally observed. It was found that the failure locus in the stress space was accurately predicted by the Von Mises criterion and failure took place by the localization of damage into a crack perpendicular to the main loading axis.
\end{abstract}

\section{Introduction}

Fibers stand for one-dimensional structural elements working under uniaxial tension. By analogy, textile materials are bidimensional arrays of fibers subjected to biaxial loads. Thus, understanding the deformation and failure mechanisms of textile materials under in-plane biaxial loading is of paramount importance for their application as structural materials. Among fabrics, nonwovens are materials manufactured from a set of disordered fibers consolidated by bonds of different nature, i.e. mechanical, thermal or chemical. They are used in a growing number of industrial applications, benefiting from the advent of high-performance fibers and improved processing machinery, including ballistic protection, fireproof layers, membranes for fuel cells, liquid-absorbing textiles, filters or geotextiles. Regarding their structural properties, nonwovens possess lower strength and stiffness than their woven counterparts, but they are superior in terms of deformation capability and energy consumption during deformation.

Despite their increasing importance, information on the mechanical behavior of nonwoven fabrics under biaxial deformation is very limited. Experimental data on the biaxial deformation of nonwovens is limited to strip band tests (in which the specimen is subjected to uniaxial strain while deformation in the perpendicular direction is constrained) and equi-biaxial tests, due to the rather complex experimental set-up required for arbitrary biaxial loading. Within this framework, Uesaka et al. [1] and Schulgasser [2] studied the elastic properties of paper while Goswami et al. [3] used biaxial tests to determine the Poisson's ratio of a thermally bonded nonwoven. Stress-strain curves for polyester and nylon 6-6 nonwovens were reported by other authors [4,5] but there is no information available on either the effect of biaxial deformation on the deformation and failure micromechanisms or the shape of failure locus.

Taking into account the experimental difficulties, numerical simulations make a most suitable tool to overcome the limitations of the testing facilities and to extend the study to arbitrarily complex biaxial loading conditions. Most of the 
work in this area has focused on woven [6-8] and knitted [9] fabrics, while very little results are available for nonwovens, which present a very different deformation pattern. They include the work of Bais-Singh and Goswami [10], based on the classical laminate theory, in which the fabric is considered to be composed of several layers, each of them containing fibers oriented along a given direction and the applied deformation is accommodated by the extension and rotation of each layer. In addition, Ridruejo et al. [11] developed a numerical model to study the deformation and failure in glass-fiber nonwoven felts under arbitrary in-plane deformation. The microstructure of the felt in the sample, made up of fiber bundles connected at the cross point through an organic binder, was explicitly included in the model. The mechanical properties of fiber bundles and interbundle bonds, as well as the characteristics of the fiber network, were obtained from independent experiments [11]. Fiber bundles were represented by Timoshenko beams, while the interbundle bonds were introduced through connectors. Failure by bond fracture and energy dissipation by friction between bundles (following experimental observations) were also taken into account. This model was successfully applied to study the deformation mechanisms and failure modes of glass-fiber nonwovens under general biaxial loading and to determine the failure locus in the stress space [12].

More recently, another model was developed to simulate the behavior of thermally-bonded polypropylene nonwoven fabrics which undergo large deformations prior to localization and failure $[13,14]$. This new model was also built within the context of the finite element method but provides the constitutive response for a mesodomain of the fabric corresponding to the area associated to a finite element instead of mimicking the actual microstructure by means of beam elements and connectors [11,12]. The model includes the effect of fiber rotation for large strains and the elasto-plastic behavior of the fiber. In addition, the various damage mechanisms experimentally observed (bond and fiber fracture) were included in a phenomenological way. It was extensively validated against experimental results [15] on smooth and notched samples subjected to uniaxial deformation and this new model is applied here to determine the failure micromechanisms and the failure locus of this nonwoven fabric under in-plane biaxial loading. Special attention was paid to study the onset and localization of damage under different loading paths, and the determination of the failure locus in the stress space.

\section{Constitutive model for the nonwoven fabric}

The constitutive model for the nonwoven fabric was developed by authors [13,14] following a detailed experimental campaign to determine the deformation and failure mechanisms of polypropylene nonwoven fabrics under in-plane loading [15]. It was found that damage began at very low strains by fracture of interfiber bonds, which caused re-arrangement of the fiber orientation. This process (bond breakage and fiber reorientation) continued upon loading, leading to a rapid reduction in the fabric stiffness and to a marked nonlinearity in the stress-strain curve. The maximum load was attained under these conditions without any evidence of fiber fracture although fibers oriented in the loading direction underwent plastic deformation. Further bond breakage resulted in the localization of damage within the fabric, leading to the development of a fracture zone of sparse fabric mainly made up of fibers aligned in the loading direction.

The constitutive model was aimed at reproducing these deformation and fracture micromechanisms and was developed within the context of the finite element method. It provides the constitutive response for a mesodomain of the fabric corresponding to the area associated to a finite element. The behavior of the fibers in the mesodomain is based on Petterson's assumptions [16] by considering a set of noninteracting straight fibers with an arbitrary initial orientation. The effect of fiber rotation for large strains and the elasto-plastic fiber behavior were rigorously taken into account by the continuum tensorial formulation. In addition, the various damage mechanisms experimentally observed (bond and fiber fracture, fiber friction and pull-out) were included in the model in a phenomenological way. Finally, the random nature of these materials as well as the changes in fiber connectivity induced by bond fracture were also included by means of a Monte Carlo lottery to determine the damage thresholds [14].

A detailed description of the constitutive model can be found elsewhere [13,14], but the main features are outlined here for the sake of clarity. The nonwoven fabric is divided into a finite number of mesodomains - which will correspond to quadrilateral finite elements in the numerical implementation. Each mesodomain contains a fiber network formed by a set of fiber families with different orientation. The constitutive model of the mesodomain is created from three blocks built on top of each other: the network model, the fiber model and the damage model. Their main features are briefly described below.

\subsection{Network model}

The deformation of the fiber network within the mesodomain is taken into account through a continuum model developed by Planas et al. [17], which constitutes a finite strain extension of the pioneering work of Cox [18]. The model considers a square planar region of arbitrary size containing a random network of long, straight, noninteracting fibers. Each fiber is characterized by a unit vector $\mathbf{N}^{\Theta}$, which forms an angle $\Theta$ with respect to a reference direction. The constitutive equation of the nonwoven fabric can be expressed as [17] 

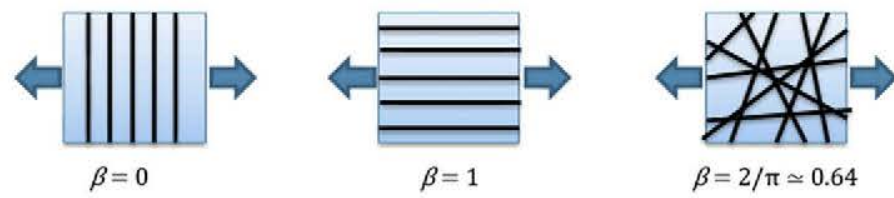

Fig. 1. Interpretation of the orientation index, $\beta$.

$$
\mathbf{S}=\frac{\rho}{\rho_{f}} \int_{-\frac{\pi}{2}}^{\frac{\pi}{2}} s_{f}^{\Theta}\left(\lambda_{f}\right) \Psi(\Theta) \frac{\left(\mathbf{N}^{\Theta} \otimes \mathbf{N}^{\Theta}\right)}{\sqrt{\mathbf{C N}^{\Theta} \cdot \mathbf{N}^{\Theta}}} \mathrm{d} \Theta
$$

where $\mathbf{S}$ stands for the second Piola-Kirchhoff stress tensor, $s_{f}^{\Theta}$ is the stress carried by the fraction of fibers oriented between $\Theta$ and $\Theta+\mathrm{d} \Theta, \lambda_{f}$ the corresponding fiber stretch, $\mathbf{C}$ the right Cauchy-Green deformation tensor of the mesodomain and $\psi(\Theta)$ the planar orientation distribution function (equal to $1 / \pi$ in the case of an isotropic fabric). $\rho$ and $\rho_{f}$ are, respectively, the nonwoven areal density and the fiber density. The ratio between both is the volume fraction of fibers per unit area of nonwoven fabric. The Cauchy stress tensor in the current (deformed) configuration is given by

$$
\sigma=\frac{1}{J} \mathbf{F S F}^{\mathrm{T}}
$$

where $J$, the Jacobian, is the determinant of $\mathbf{F}$.

Another important outcome of the model is the degree of orientation of the fibers within the fabric, characterized by the orientation index $\beta$,

$$
\beta=\int_{-\frac{\pi}{2}}^{\frac{\pi}{2}} \frac{\mathbf{F N}^{\Theta}}{\left\|\mathbf{F} \mathbf{N}^{\Theta}\right\|} \cdot \mathbf{e}_{1} \Psi(\Theta) \mathrm{d} \Theta
$$

where $\mathbf{e}_{1}$ is the unit vector along a reference direction. According to this definition, $\beta$ is comprised in the interval $[0,1]$, where $\beta=0$ implies that all fibers are oriented perpendicular to the privileged direction $\mathbf{e}_{1}$ and $\beta=1$ implies that all the fibers are parallel to $\mathbf{e}_{1}$. A perfectly isotropic fiber distribution is characterized by $\beta=2 / \pi \approx 0.64$ (Fig. 1).

\subsection{Fiber model}

The influence of the fiber properties on the mechanical performance of the fabric is introduced in the constitutive model through the nominal stress - stretch $s_{f}\left(\lambda_{f}\right)$ function. Following experimental tests on individual fibers extracted from the nonwoven fabric, the fiber behavior was accurately represented by a one-dimensional elastic-plastic model [15]. The total engineering strain was decomposed into the sum of an elastic (reversible) component, $e_{f}^{e}$ and a plastic (permanent) one, $e_{f}^{p}$,

$$
e_{f}=e_{f}^{e}+e_{f}^{p}
$$

The one-dimensional, rate-independent plastic behavior with isotropic hardening was formulated according to Simó and Hughes [19] and the constitutive response excluding damage of an individual fiber is summarized in Fig. 2. Compressive stresses were not allowed in fibers since they buckle at very low loads. The elastic strains were related to the fiber stresses through the fiber elastic modulus

$$
s_{f}=E_{f} e_{f}=E_{f}\left(\lambda_{f}^{e}-1\right)
$$

while plastic deformation is controlled by the yield function $\Phi$ given by

$$
\Phi=s_{f}-\left(s_{f, y}+H_{f} \bar{e}_{f}^{p}\right)
$$

where $s_{f}^{y}$ and $H_{f}$ stand, respectively, for the fiber yield strength and the hardening modulus and $\bar{e}_{f}^{p}$ is the accumulated plastic strain, which is computed as

$$
\bar{e}_{f}^{p}=\int_{0}^{t} \dot{e}_{f}^{p} \mathrm{~d} t
$$

The density and the mechanical properties of the fibers were obtained from tests on individual fibers extracted from the fabric [15] and can be found in Table 1 . In addition, the fabric areal density $\rho\left(118 \mathrm{~g} / \mathrm{m}^{2}\right)$ was also measured independently [15]. 


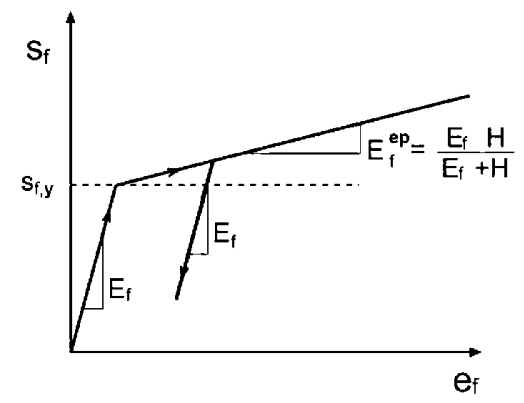

Fig. 2. Elastic-plastic behavior of a polypropylene fiber.

Table 1

Density and parameters of the engineering stress-engineering strain curve of polypropylene fibers extracted from the nonwoven fabric.

\begin{tabular}{llllll}
\hline$\rho_{f}\left(\mathrm{~g} / \mathrm{cm}^{3}\right)$ & $E_{f}(\mathrm{GPa})$ & $s_{f, y}(\mathrm{MPa})$ & $H_{f}(\mathrm{MPa})$ & $s_{f}^{u}(\mathrm{MPa})$ & $e_{f}^{u}$ \\
\hline 0.91 & $1.7 \pm 0.1$ & $120 \pm 4$ & $59 \pm 2$ & $240 \pm 10$ & $1.4 \pm 0.1$ \\
\hline
\end{tabular}

Table 2

Parameters controlling the onset and propagation of damage in the nonwoven fabric.

\begin{tabular}{llll}
\hline$b_{0}(\mathrm{MPa})$ & $B(\mathrm{MPa})$ & $m$ & $G_{F}\left(\mathrm{~kJ} / \mathrm{m}^{2}\right)$ \\
\hline 20 & 360 & 3.6 & 550
\end{tabular}

\subsection{Damage model}

The constitutive model for the mesodomain is completed with a damage model. Damage in thermally-consolidated nonwoven polypropylene fabrics is mainly triggered by interfiber bond fracture. The first effect of bond fracture is to reduce the load carried by the fibers crossing at the broken bond, but it also changes the connectivity of the network. Thus, further deformation gives rise to a re-arrangement of the fiber network accompanied by extensive fiber rotation, and fibers are eventually reloaded as they become aligned to the main loading axis. Finally, fiber fracture occurs in the last stages of deformation well beyond the peak-load in the stress-strain curve [15].

It is evident that these mechanisms, involving changes in the topology of the fiber network, cannot be explicitly accounted for in a continuum model like the one presented here and a phenomenological approach was adopted $[13,14]$. The reduction in the stress carried by the fibers as a result of bond fracture and of fiber failure was taken into through a standard uni-dimensional continuum damage model in the fiber constitutive equation [20]. Thus, the stress carried by the fiber decreased exponentially after the onset of the damage and the area under the stress-strain curve stands for the energy dissipated during fracture per unit volume of fibers, $g_{f} . g_{f}$ includes the energy dissipated during fiber deformation and the contributions due to frictional sliding between fibers after bond fracture and to fiber pull-out after fiber fracture.

However, fibers can be reloaded at later stages as the fabric deforms and fibers undergo large rotations. One simple way to include this effect into the continuum damage model is to assume that the parameter $b$ (which controls the onset of damage in each fiber) is not constant but varies during deformation. Thus, $b$ was assumed to follow a Weibull distribution and its value was computed at each time increment during the simulation using a Monte Carlo lottery according to

$$
b=B[-\ln (1-p)]^{1 / m}+b_{0}
$$

where $p$ is a random number in the range $|0,1|, b_{0}$ a threshold stress for damage and $B$ and $m$ stand for the two parameters of the Weibull distribution. Regardless of the statistical distribution, it was assumed that $b<s_{f}^{u}$, the fiber tensile strength. In each loading step, if $b$ was below the current stress carried by the fibers, the reduction in the load carried by the fiber was determined according to the uni-dimensional continuum damage model. Otherwise, the fiber was reloaded, following the elasto-plastic fiber model presented in the previous section (although the fiber elastic modulus was reduced according to the amount of damage undergone by the fiber up to that stage). More details about the actual damage model are outside the scope of this article and can be found in [13,14].

Neither the fiber fracture energy nor the parameters of the Weibull model to determine the onset of damage could be measured experimentally, and they were obtained by fitting the results of the numerical simulations to the experimental stress-strain curve of the nonwoven fabric under tensile deformation [14]. The corresponding values can be found in Table 2 . 


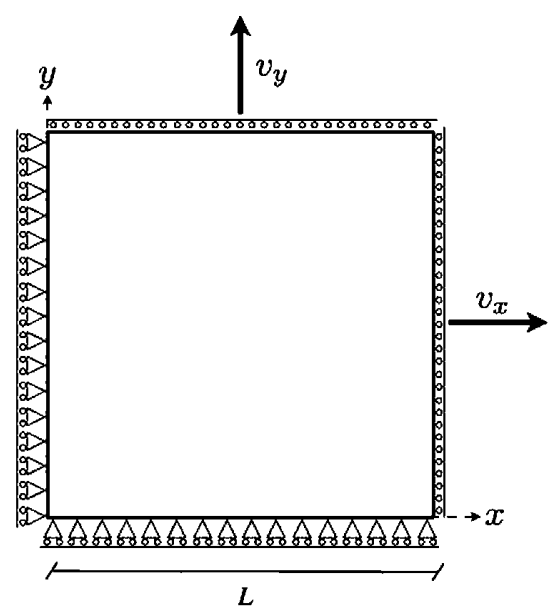

Fig. 3. Schematic of the boundary conditions for biaxial deformation.

\subsection{Numerical model}

The constitutive model for the mesodomain was implemented in Abaqus Explicit (v6.8) as a user material subroutine (VUMAT). Simulations were carried out under plane stress conditions within the framework of quasi-static loading and large displacements and rotations. The equations of motion for the body were integrated using an explicit central difference integration rule together with the use of diagonal ("lumped") element mass matrices. More details can be found in Refs. [14] and [21].

Square specimens of $100 \times 100 \mathrm{~mm}^{2}$ were discretized with $40 \times 40$ CPS4R bilinear plane stress elements, with reduced integration (one Gauss point per element) and hourglass control. Each finite element was considered as a isotropic mesodomain made up of fifty sets of fibers oriented in different directions. The angular integration of the constitutive law for the mesodomain (Eq. (1)) was performed by using Simpson's rule running over 50 subintervals corresponding to different fiber orientations.

Different boundary conditions were applied to the square specimens to simulate the experimental tests under tension and to compute the mechanical behavior under biaxial loading. In the former case, the displacements on the upper and lower boundaries were prescribed in both directions according to

$$
\begin{aligned}
& \dot{\mathbf{u}}(0, y)=0, \quad \dot{u}_{x}(L, y)=v_{x}, \quad \dot{u}_{y}(L, y)=0 \\
& \mathbf{T}(x, 0)=\mathbf{T}(x, L)=0, \quad y \neq 0, \quad y \neq L
\end{aligned}
$$

where $\mathbf{u}$ and $\mathbf{T}$ stand, respectively, for the displacement and tractions applied to the specimen boundary and $v_{x}=20 \mathrm{~mm} / \mathrm{s}$ was the applied velocity on the upper boundary of the cell. This velocity was higher than the experimental one [15] to reduce the overall computing time. It should be noted, however, that quasi-static loading conditions were maintained as the kinetic energy in the model was negligible as compared to the strain energy.

Biaxial deformation on the square specimen was obtained with the following set of boundary conditions (Fig. 3 )

$$
\begin{aligned}
& \dot{u}_{y}(x, 0)=0 \quad \text { and } \quad \dot{u}_{y}(x, L)=v_{y} \\
& \dot{u}_{x}(0, y)=0 \quad \text { and } \dot{u}_{x}(L, y)=v_{x} \\
& T_{x}(x, 0)=T_{x}(x, L)=0, \quad x \neq 0, x \neq L \\
& T_{y}(0, y)=T_{y}(L, y)=0, \quad y \neq 0, \quad y \neq L
\end{aligned}
$$

where $v_{x}$ and $v_{y}$ stand for the applied velocities on the specimen boundaries. $v=20 \mathrm{~mm} / \mathrm{s}$ was the maximum applied velocity on the boundary of the cell. In addition to biaxial deformation, simulations under uniaxial tension (in which the edges perpendicular to the loading axis were stress-free) were also carried out with the following boundary conditions:

$$
\begin{aligned}
& \dot{u}_{x}(0, y)=0 \quad \text { and } \quad \dot{u}_{x}(L, y)=v_{x} \\
& T_{y}(0, y)=T_{y}(L, y)=0 \\
& \mathbf{T}(x, 0)=\mathbf{T}(x, L)=0, \quad x \neq 0, x \neq L
\end{aligned}
$$

It is known that the damage models including softening lead to mesh-dependent results because the energy dissipated is a function of the element size. In order to overcome this limitation, the numerical model was regularized by means 


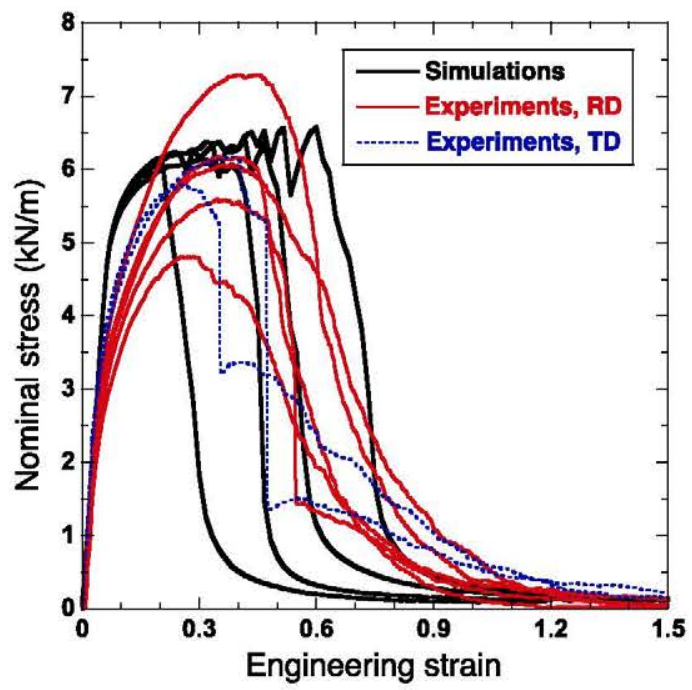

Fig.4. Numerical and experimental stress-strain curves of square specimens $\left(100 \times 100 \mathrm{~mm}^{2}\right)$ subjected tensile loading along the rolling (RD) and transverse (TD) directions.

of the crack band approach [22,23] so the energy dissipated in each element during loading, $G_{F}$, was independent of the refinement of the mesh. Mathematically, this condition is introduced as

$$
G_{F}=g_{f} l_{c h}
$$

where $l_{c h}$ was equal to the square root of the finite element area.

Finally, local instabilities in the form of high frequency vibrations may lead to impaired convergence. They were eliminated by using bulk viscosity which introduces the pressure term $P_{v}$ associated with the volumetric strain rate according to

$$
P_{v}=b \rho c_{d} L_{d} \dot{\epsilon}_{v o l}
$$

where $b$ is a damping coefficient $(b=0.2), \rho$ the fabric density and $\dot{\epsilon}_{v o l}$ the volumetric strain rate. This viscous pressure term damps the high frequency oscillations and for this reason it is also known as "truncation frequency damping". Bulk viscosity is also internally used for hourglass control. The presence of damping penalizes the stable time increment to some extent [24], but it proves useful in order to ensure accurate dynamic modeling [21].

\section{Experimental validation}

Square specimens of $100 \times 100 \mathrm{~mm}^{2}$ of a polypropylene nonwoven fabric were tested in tension. Samples were oriented along the rolling (RD) and transverse (TD) directions of the fabric. The ends of the specimens were clamped between two steel plates protected with rubber to avoid cutting the fabric. Tests were carried out under stroke control at $0.8 \mathrm{~mm} / \mathrm{min}$, and load and cross-head displacement were continuously recorded during the test. In addition, the deformation pattern on the specimen surface was obtained by means of digital image correlation using commercial software (VIC 2D) [25]. More details about the experimental set-up can be found elsewhere [15].

The experimental values of the nominal tensile stress (obtained as the total force divided by the initial width) vs. the applied engineering strain (obtained as the cross-head displacement divided by the initial length) are plotted in Fig. 4. They include five tests carried out along the rolling direction (RD) of the fabric and two along the transverse direction (TD). The behavior under both directions was equivalent as a result of the isotropic orientation of the fibers in the nonwoven felt. After a short linear region, the curves presented a marked nonlinear behavior and the maximum load carrying capacity was attained at engineering strains in the range $25-45 \%$. Afterwards, two different post-peak behaviors were found. The first one was characterized by a gradual reduction of the stress borne by the fabric while the second presented one (or, sometimes, two) abrupt reductions in stress, associated to the sudden localization of damage, followed by a slight reloading as a result of fiber re-arrangement. Both set of curves presented a long tail as the stresses carried by the fabric were reduced to zero at engineering strains above $100 \%$. The scatter among the tests was significant, particularly regarding the maximum load carrying capacity and the strain at the onset of damage localization.

The shape of the deformed fabric is shown in Fig. 5. The specimens presented the typical hourglass shape due to the constrained horizontal deformation at the ends and deformation was homogeneous prior to the onset of damage localization (Fig. 5(a)). Damage localized in a band perpendicular to the loading axis near to center of the specimen (Fig. 5(b)) in which the residual strength of the fabric was basically provided by the surviving aligned fibers in an increasingly sparse felt. The 

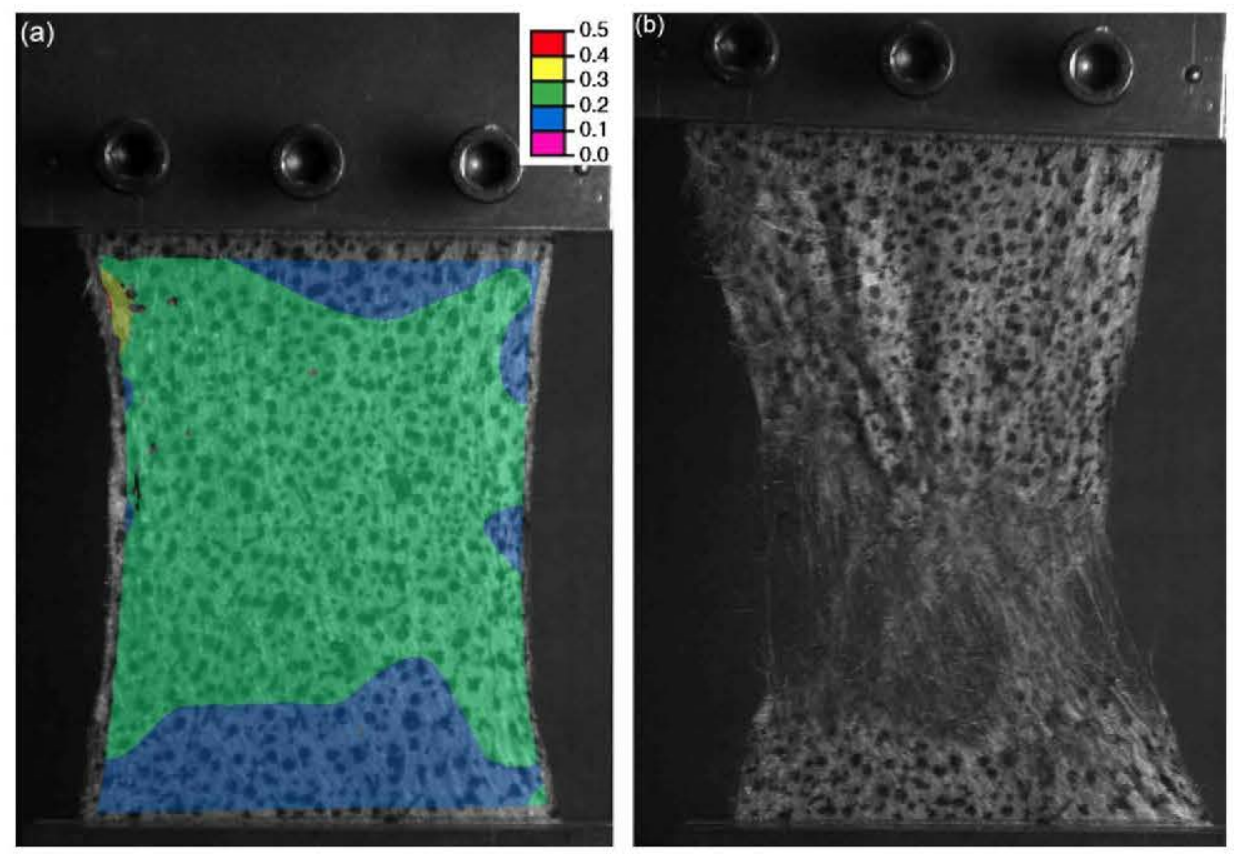

Fig. 5. (a) Contour plot of the Lagrangian strain $\epsilon_{y y}$ along the loading direction as obtained by digital image correlation at an applied engineering strain of $21 \%$. (b) Localization of damage along the horizontal band. The nominal applied strain was $37 \%$,

precise region and the critical applied strain for damage localization was very likely dictated by the fabric "cloudiness" i.e., density fluctuations - and changed significantly among the specimens.

The nominal stress-strain curves obtained from the numerical simulations are also plotted in Fig. 4(a) for comparison. All the simulations were carried out with identical properties for the nonwoven fabric, obtained from [15,14], and the differences among the curves are due to the Monte Carlo damage model. The simulated curves slightly underestimated the nonlinearity prior to the maximum load but very accurately captured the elastic modulus, the average value of the nominal strength, the strain at the onset of damage localization (including the actual scatter in this magnitude) and the tail of the curve after localization. The model also predicted the formation of a damage band in which the surviving fibers were aligned parallel to the loading axis (not plotted for the sake of brevity).

\section{Failure locus under biaxial loading}

The constitutive model for the nonwoven fabric was used to obtain the failure locus in the $S_{x}-S_{y}$ nominal stress space. The square specimens were subjected to uniaxial tension and biaxial deformation in the $x y$ plane. In the case of the biaxial simulations, different loading paths (characterized by the ratio $v_{y} / v_{x}$ ) were used.

The results of the numerical simulations corresponding to one fiber network are plotted in Figs. 6 to 10. They correspond to uniaxial tension (Fig. 6), uniaxial deformation ( $v_{y}=0$, Fig. 7), biaxial deformation $\left(v_{y}=0.25 v_{x}\right.$, Fig. 8 and $v_{y}=0.5 v_{x}$, Fig. 9) and equi-biaxial deformation ( $v_{y}=v_{x}$, Fig. 10). Each figure presents the nominal tensile stresses along both directions, $S_{x}$ and $S_{y}$ (obtained as the total normal force acting on the specimen boundary divided by the initial length $L$ ) as a function of the corresponding applied engineering strains $\epsilon_{x}\left(=u_{x} / L\right)$ and $\epsilon_{y}\left(=u_{y} / L\right)$. In addition, two contour plots are provided to understand the dominant failure mechanisms in the nonwoven fabric as a function of the stress state. The first one corresponds to the average damage variable, $D$, for each mesodomain (i.e. finite element) after failure has localized. $D$ is obtained by averaging the damage variables of all the fibers in each mesodomain. Elements with $D>0.98$ are deleted from the numerical model but those with $D>0.94$ were not displayed in the contour plots for the sake of clarity. The second contour plot represents the fiber orientation index $\beta$ at the final stages of deformation.

\subsection{Uniaxial stress}

The simulation results for the specimen loaded under uniaxial tension are shown in Fig. 6. The nominal stress-strain curve (Fig. 6(a)) presented the same features reported in the previous section to validate the experimental results, as the only difference between both simulations was that the displacement perpendicular to the loading direction was not constrained at the specimen ends. The initial linear elastic response was followed by a nonlinear zone due to the homogeneous nucleation of damage throughout the specimen by interbond fracture. This process continued until a maximum in the load carrying capability was achieved (at engineering strains of the order of $30-40 \%$ ), and it was followed shortly afterwards by 


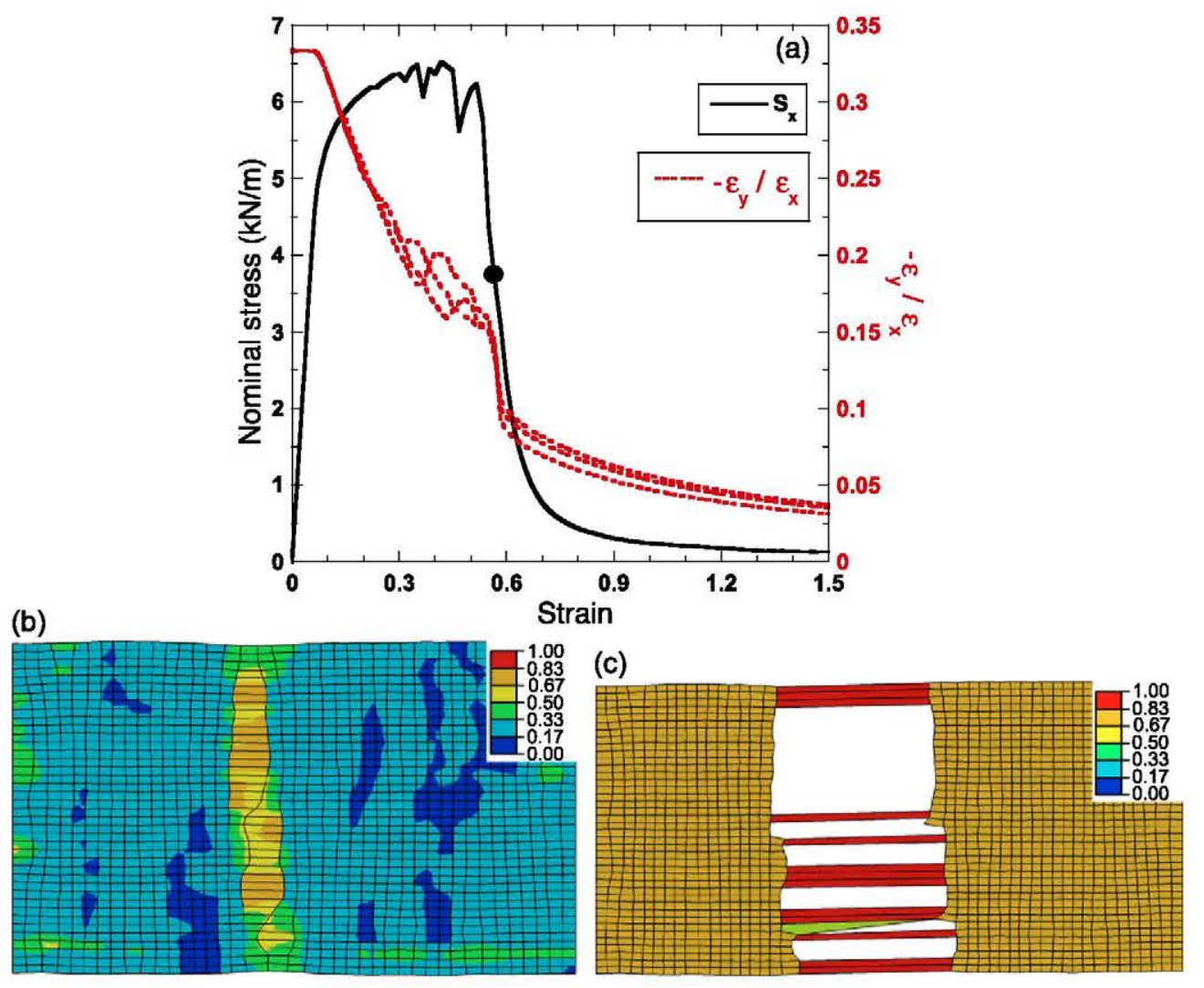

Fig. 6. (a) Nominal stress $\left(S_{x}\right)$ vs. engineering strain $\left(\epsilon_{x}\right)$ curve of the nonwoven fabric deformed under uniaxial tension. (b) Contour plot of the average damage, $D$, at the point marked with a circle in the $S_{x}-\epsilon_{x}$ curve in (a). (c) Contour plot of the fiber orientation index, $\beta$, after damage localization. $\beta=1$ indicates that all the fibers were oriented along the horizontal $x$ axis.

the localization of damage. The serrated shape of the $S_{x}-\epsilon_{x}$ curve prior to damage localization shows the phenomenological model's ability to simulate the reloading of the nonwoven by the re-arrangement of the fiber network. Damage was distributed homogeneously throughout the specimen until it was localized in a band perpendicular to the loading axis at applied strains of $\approx 50 \%$ (Fig. $6(\mathrm{~b})$ ). The load was not reduced to zero after the localization of damage and the curves presented a long tail associated to the load transferred by a limited number of fibers oriented parallel to the loading axis (Fig. 6(c)).

The uniaxial tension tests also provide information about the evolution of the Poisson's ratio $\left(-\epsilon_{y} / \epsilon_{x}\right)$ during deformation, which is plotted in Fig. 6(a). The three curves in this figure correspond to the Poisson's ratio in different positions along the specimen located at the specimen ends and at the failure section and the differences among them were minor. The initial value in the elastic regime (0.33) is in agreement with the predictions of Cox's model for a random network of long, elastic, noninteracting fibers. Finite deformations and damage lead to a continuous reduction in the Poisson's ratio upon deformation, which were reduced sharply at the onset of damage localization, leading to values close to 0 in the final stages.

\subsection{Uniaxial strain}

Uniaxial strain tests (also named strip band tests) were carried out by impeding the deformation of the specimen perpendicular to the loading axis, leading to the development of stresses perpendicular to the loading direction. The evolution of nominal stresses in both directions $\left(S_{x}\right.$ and $\left.S_{y}\right)$ as a function of the applied engineering strain $\left(\epsilon_{x}\right)$ is plotted in Fig. 7(a). The lateral constraint under uniaxial deformation (which led to the development of significant stresses perpendicular to the lading axis) induced changes in the nominal stress-strain curve as compared with uniaxial tension. Basically, the maximum nominal stresses carried by the fabric and the applied strain at the onset of localization were higher than those obtained under uniaxial tension. The shape of the stress-strain curve was also modified, passing from a bell shape to a more serrated, trapezoidal one. 


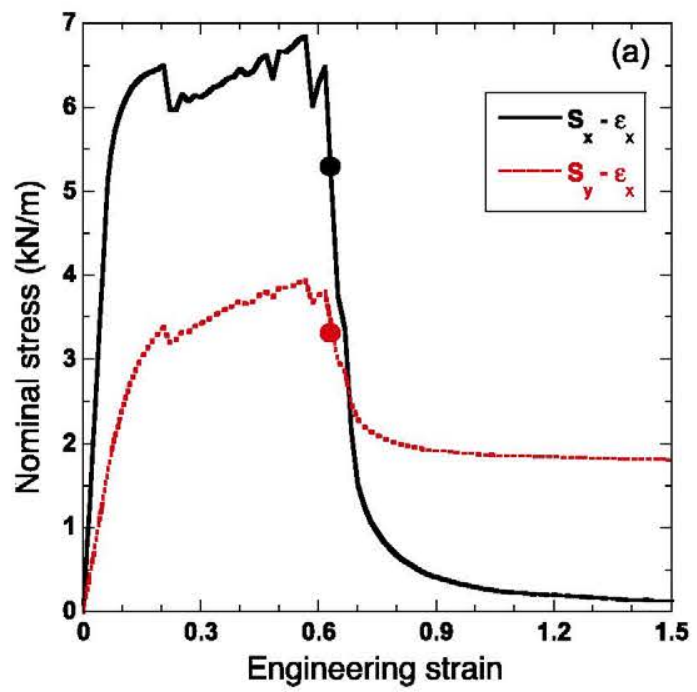

(b)

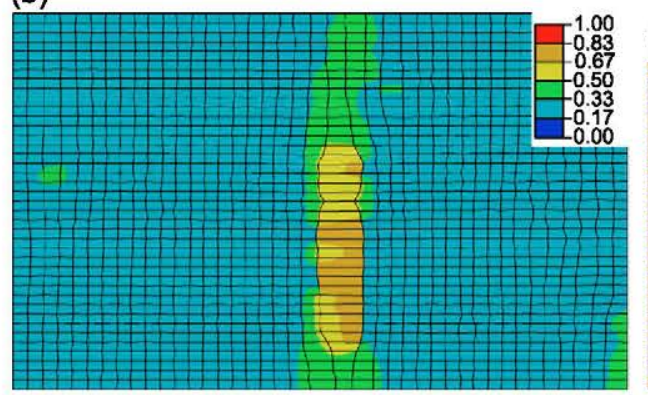

(c)

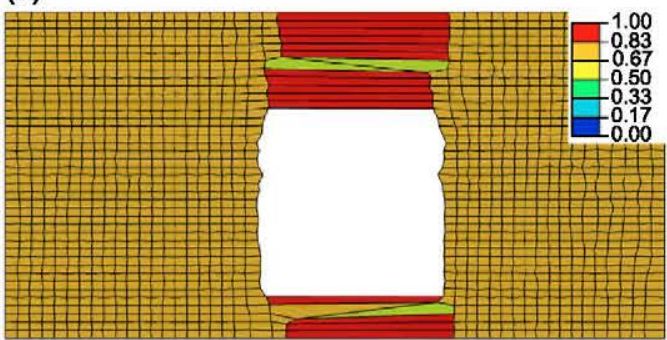

Fig. 7. (a) Evolution of nominal stresses in both directions ( $S_{x}$ and $\left.S_{y}\right)$ as a function of the applied engineering strain $\left(\epsilon_{x}\right)$ in the nonwoven fabric deformed under uniaxial deformation along the $x$ direction. (b) Contour plot of the average damage, $D$, at the point marked with a circle in the stress-strain curves in (a). (c) Contour plot of the fiber orientation index, $\beta$, after damage localization. $\beta=1$ indicates that all the fibers were oriented along the horizontal $x$ axis.

\subsection{Biaxial deformation}

Simulations with different amounts of biaxial stresses were carried out by applying different velocities $v_{x}$ and $v_{y}$ on the specimen boundaries. The results for the cases $v_{y}=0.25 v_{x}$ and $v_{y}=0.5 v_{x}$ are shown in Figs. 8 and 9. The evolution of nominal stresses in both directions $\left(S_{x}\right.$ and $\left.S_{y}\right)$ as a function of the applied engineering strains $\left(\epsilon_{x}\right.$ and $\left.\epsilon_{y}\right)$ are plotted in Figs. 8(a) and 9(a). Biaxial deformation changes the shape of the stress-strain curve along the main loading axis $(x)$, as compared with the uniaxial tension test. Stress is almost linear with strain until a horizontal plateau is reached and the bell shape found under uniaxial tension (Fig. 6(a)) has disappeared. This is probably a consequence of the constraint imposed on fiber rotation by the boundary conditions, which limits the nonlinear contribution of fiber rotation to the strain. In addition, the response of the fabric is stiffer in the linear regime along the axis deformed at slower velocity ( $y$ in this case).

When the deformation rate in one direction was much higher than in the perpendicular one, damage localized perpendicular to the axis deformed at higher velocity (Figs. 8(b) and 8(c)). The load carrying capability along the perpendicular axis was reduced at the onset of strain localization but it increased again once the damage zone was fully developed, indicating that the fabric was fully capable of taking up more load outside of the damage region. However, damage may nucleate along the axis deformed at slower velocity if the differences between $v_{x}$ and $v_{y}$ were smaller (Fig. 9(b)). Nevertheless, final fracture always took place by the development of a crack (bridged by a limited number of fibers) perpendicular to the main loading axis (Fig. 9(c)) and the localization of damage perpendicular to the secondary loading axis (although could start earlier) only was completely developed after the fabric has lost all the load carrying capacity along the main loading axis.

\subsection{Equi-biaxial deformation}

In the case of equi-biaxial deformation (Fig. 10(a)), the elastic moduli were identical in both directions and the differences in maximum load carrying capability in both directions were minimum. In addition, the strength was very similar 


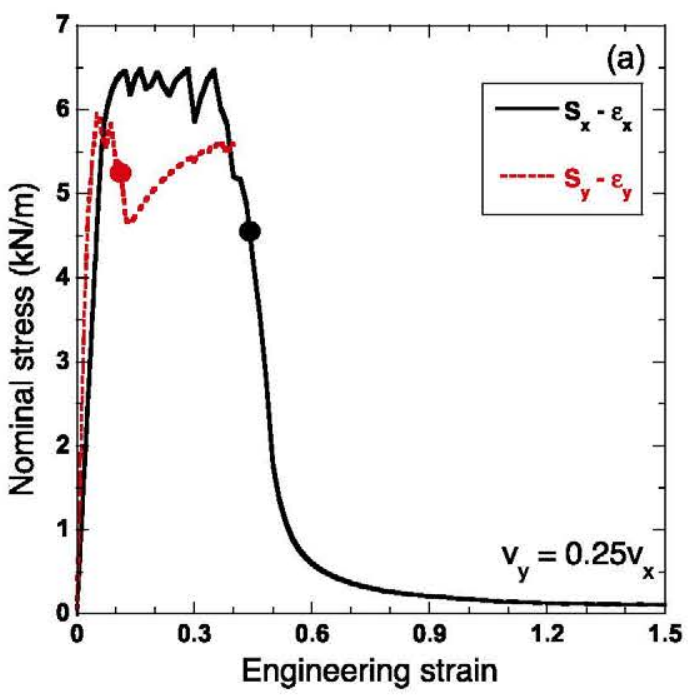

(b)
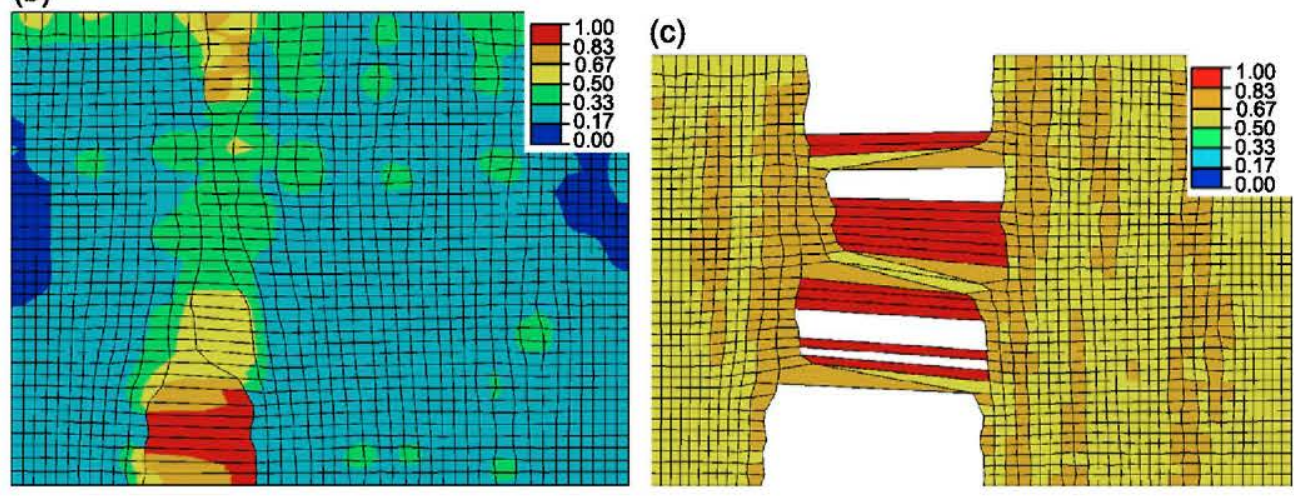

Fig. 8. (a) Evolution of nominal stresses in both directions $\left(S_{x}\right.$ and $\left.S_{y}\right)$ as a function of the applied engineering strains $\left(\epsilon_{x}\right.$ and $\left.\epsilon_{x}\right)$ in the nonwoven fabric deformed with $v_{y}=0.25 v_{x}$. (b) Contour plot of the average damage, $D$ at the points marked with circles in the $S_{x}-\epsilon_{x}$ and $S_{y}-\epsilon_{y}$ curves in (a). (c) Contour plot of the fiber orientation index, $\beta$, after damage localization. $\beta=1$ indicates that all the fiber are aligned parallel to the horizontal $x$ axis.

to the one obtained under uniaxial tension but it was attained at lower strains (10\%) and the transition from the linear region to the peak has almost disappeared. This behavior is again induced by the constraint imposed on fiber rotation by the equi-biaxial boundary conditions.

The localization pattern was different from those reported above. Final fracture occurred through the development of two damage bands parallel to the $x$ and $y$ axis, respectively (Figs. $10(\mathrm{~b})$ and $10(\mathrm{c})$ ). Although the onset of damage was almost simultaneous in both bands (Fig. 10(a)), localization developed faster in the band parallel to the $x$ axis. Once the crack band perpendicular to the $y$ axis was fully developed $\left(\epsilon_{y} \approx 40 \%\right)$ and the fabric had lost its load carrying capability along the $y$ axis, localization began perpendicular to the $x$ axis until another crack band was developed.

\subsection{Failure locus}

The uniaxial and biaxial simulations presented above can be used to determine the failure locus of the polypropylene nonwoven felt in the $S_{x}-S_{y}$ stress space. The strength in both directions under uniaxial tension, uniaxial deformation and various biaxial loading paths was obtained and the corresponding data are plotted in Fig. 11. One or two simulations were carried out for each loading path to account for variability in the results induced by the damage model but the differences in strength were limited. The failure locus predicted by the Von Mises criterion was also plotted for the sake of comparison. The Von Mises criterion states that the failure locus is dictated by a critical value of the second deviatoric stress invariant $J_{2}$. This can be expressed as

$$
S_{x}^{2}-S_{x} S_{y}+S_{y}^{2}=S_{u n i a x i a l}^{2}
$$

under plane stress conditions where $S_{\text {uniaxial }}$ stands for the nominal strength under uniaxial tension. As shown in Fig. 11, this criterion accurately follows the failure surface for this polypropylene nonwoven and it was able to predict that the 


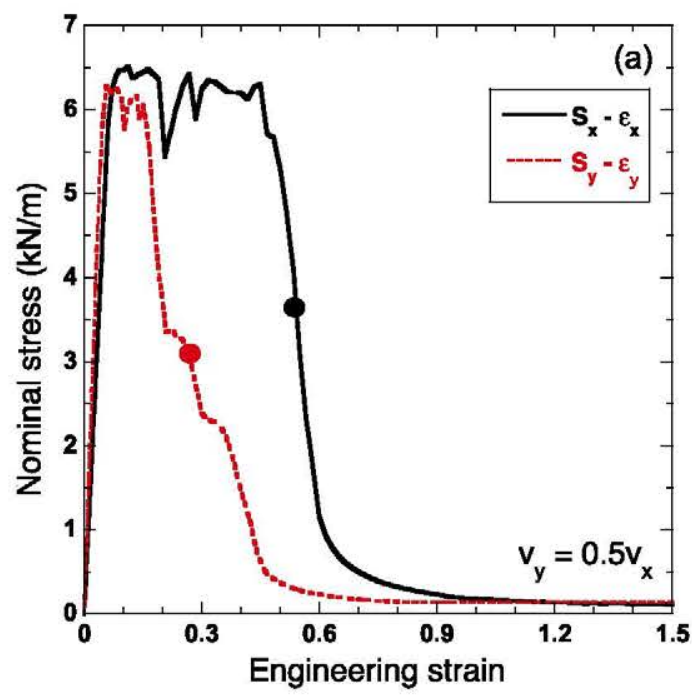

(b)

(c)
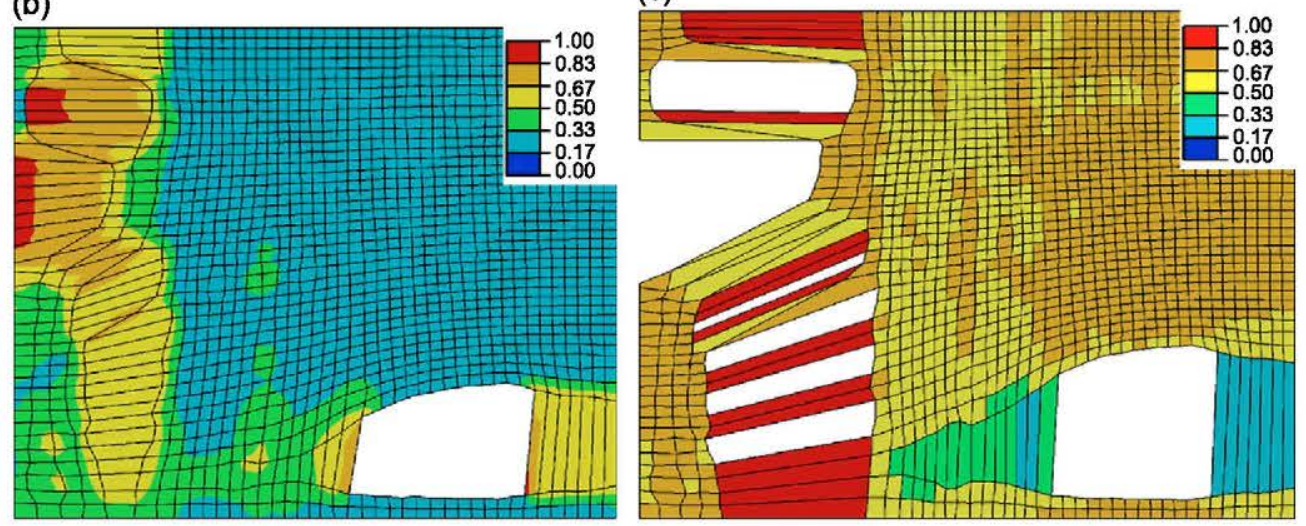

Fig. 9. (a) Evolution of nominal stresses in both directions $\left(S_{x}\right.$ and $\left.S_{y}\right)$ as a function of the applied engineering strains $\left(\epsilon_{x}\right.$ and $\left.\epsilon_{x}\right)$ in the nonwoven fabric deformed with $v_{y}=0.5 v_{x}$. (b) Contour plot of the average damage, $D$, at the points marked with circles in the $S_{x}-\epsilon_{x}$ and $S_{y}-\epsilon_{y}$ curves in (a). (c) Contour plot of the fiber orientation index, $\beta$, after damage localization. $\beta=1$ indicates that all the fiber are aligned parallel to the horizontal $x$ axis.

maximum load-carrying capacity of the nonwoven was attained under uniaxial deformation. Either uniaxial tension (in which the deformation perpendicular to the loading direction is unconstrained) or any biaxial loading path led to slightly lower strength values. In addition, the applied strain at the onset of localization was also maximum in the specimens loaded in uniaxial deformation. Although the physical basis for this fit is not obvious, it is worth noticing that the Von Mises criterion was also found to provide the best agreement with the predicted behavior of a glass-fiber nonwoven fabric [12], whose overall mechanical behavior was very different from the polypropylene fabrics studied here.

\section{Conclusions}

The mechanical behavior of a commercially-available nonwoven fabric made of polypropylene under in-plane biaxial loading was studied by means of a homogenization model. The model was developed within the context of the finite element method and provides the constitutive response for a mesodomain of the fabric corresponding to the area associated to a finite element. The mesodomain is made up of a set of fibers with different orientation and the model explicitly and rigorously includes the effects of fiber rotation and of the elastic-plastic deformation of polypropylene fibers. Additionally, the model accounts for interfiber bond fracture by means of a phenomenological approach based on a Monte Carlo lottery. All the model parameters were obtained from experimental results [15] excepting those corresponding to the onset and spread of damage, which were determined by fitting the overall model predictions to the experimental uniaxial stressstrain curves $[13,14]$.

Under uniaxial tension, the nominal stress-strain curve of the nonwoven fabric presented a short linear region, which was followed by a nonlinear zone which led to a plateau at which the maximum load carrying capability was attained 


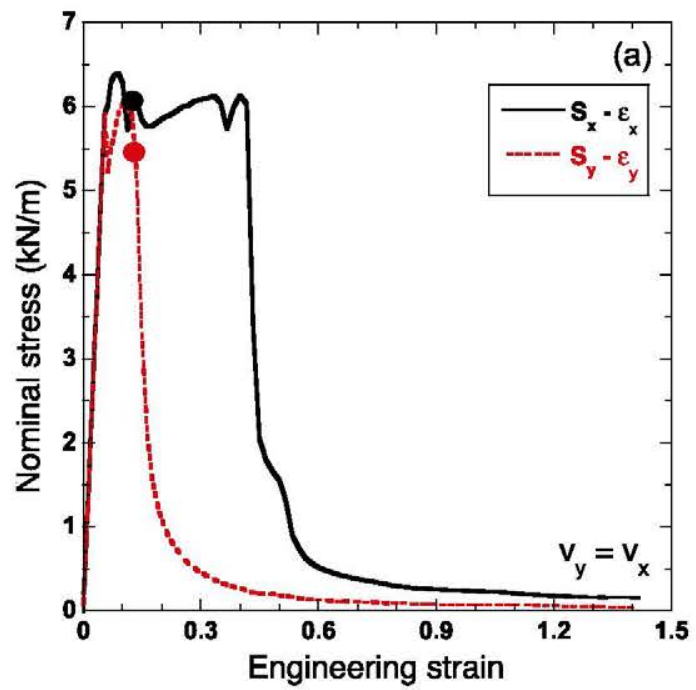

(b)

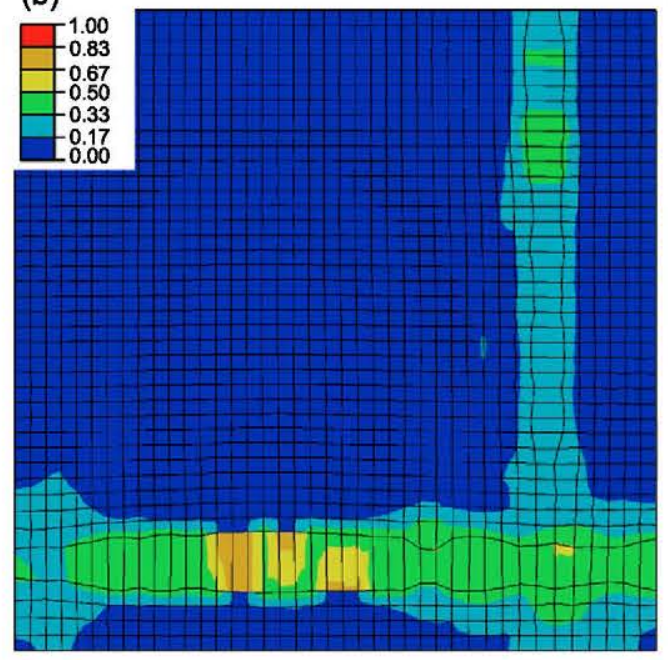

(c)

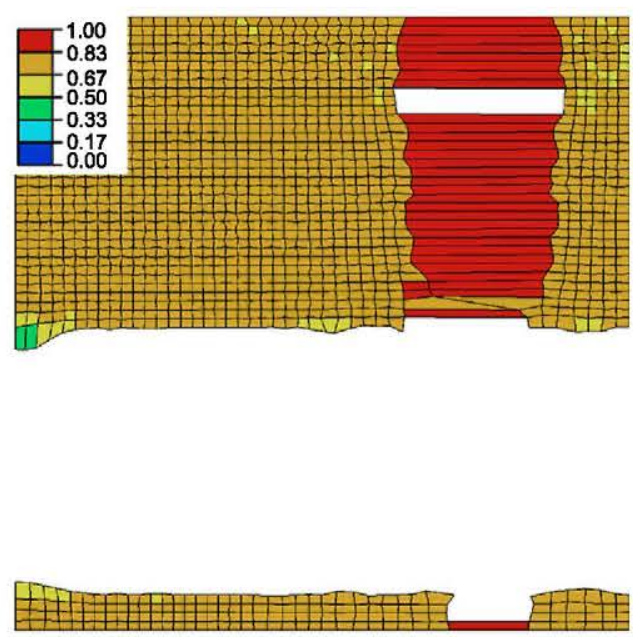

Fig. 10. (a) Evolution of nominal stresses in both directions $\left(S_{x}\right.$ and $\left.S_{y}\right)$ as a function of the applied engineering strains ( $\epsilon_{x}$ and $\left.\epsilon_{x}\right)$ in the nonwoven fabric deformed under with equi-biaxial deformation. (b) Contour plot of the average damage, $D$, at the onset of strain localization marked with a circle in the $S_{x}-\epsilon_{x}$ and $S_{y}-\epsilon_{y}$ curves in (a). (c) Contour plot of the fiber orientation index, $\beta$, after damage localization. $\beta=1$ indicates that all the fiber are aligned parallel to the horizontal $x$ axis.

(at engineering strains of the order of 30\%-40\%). Damage localization in a band perpendicular to the loading axis occurred shortly afterwards and the nominal stress-strain curve presented a long tail as the stresses carried by the fabric were reduced to zero at engineering strains above $100 \%$. The nominal stress-strain curves under biaxial deformation presented similar features although the linear region was expanded and the nonlinear zone reduced, leading to a sharp transition between the elastic regime and the plateau region. Final fracture always took place by the development of a crack (bridged by a limited number of fibers) perpendicular to the main loading axis. The localization of damage perpendicular to the secondary loading axis only occurred after the fabric had lost all the load carrying capacity along the main loading axis. Finally, the failure locus of the nonwoven fabric under in-plane biaxial loading closely followed the Von Mises criterion in the stress space.

\section{Acknowledgements}

This investigation was supported by the Spanish Ministry of Science and Innovation through grants MAT2009-14396 and MAT2009-13979 and by the Comunidad de Madrid (Regional Government of Madrid) through the program ESTRUMAT (S-2009/MAT-1585). 


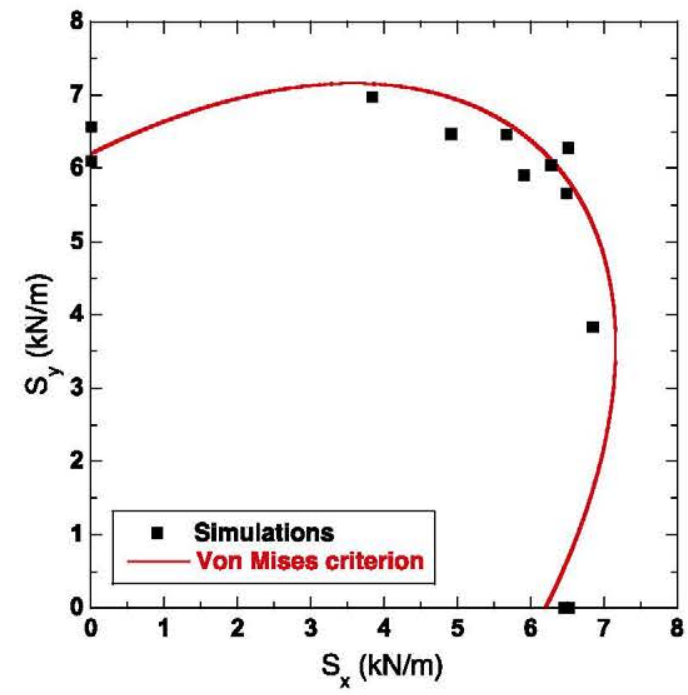

Fig. 11. Failure locus in the stress space for the polypropylene nonwoven. The predictions of the Von Mises criterion are also plotted for comparison.

\section{References}

[1] T. Uesaka, K. Murakami, R. Imamura, Biaxial tensile behavior of paper, Tappi Journal 62 (8) (1979) 111-114.

[2] K. Schulgasser, The in-plane Poisson ratio of paper, Fibre Science and Technology 19 (4) (1983) 297-309.

[3] B.C. Goswami, J. Suryadevara, T.L. Vigo, Determination of Poisson's ratio in thermally bonded nonwoven fabrics, Textile Research Journal 54 (1984) 391-396.

[4] K. Matsumoto, R. Fukae, N. Uchino, N. Utsumi, Uniaxial and biaxial tensile properties of nonwoven fabrics. I. On the toughness of nonwoven fabrics, Journal of the Textile Machinery Society of Japan 37 (1984) 19-29.

[5] H.J. Barndt, J.R. Wagner, Biaxial tensile evaluation of resin bonded nonwoven webs, in: Nonwovens, Symposium Notes of the Technical Association of the Pulp and Paper Industry, 1988, pp. 183-194.

[6] K. Buet-Gautier, P. Boisse, Experimental analysis and modeling of biaxial mechanical behavior of woven composite reinforcements, Experimental Me chanics 41 (2001) 260-269.

[7] P. Boisse, M. Borr, Experimental study of biaxial mechanical behaviour in fabrics, Comptes Rendus - Académie des Sciences, Série II 323 (1996) $503-$ 509.

[8] C. Galliot, R.H. Luchsinger, A simple model describing the non-linear biaxial tensile behaviour of PVC-coated polyester fabrics for use in finite element analysis, Composite Structures 90 (2009) 438-447.

[9] Y. Luo, I. Verpoest, Biaxial tension and ultimate deformation of knitted fabric reinforcements, Composites - Part A: Applied Science and Manufacturing 33 (2002) 197-203.

[10] S. Bais-Singh, B.C. Goswami, Predicting the biaxial tensile deformation behavior of spunbonded nonwovens, Textile Research Journal 68 (1998) $219-227$.

[11] A. Ridruejo, C. González, J. Llorca, Damage micromechanisms and notch sensitivity of glass-fiber non-woven felts: an experimental and numerical study, Journal of the Mechanics and Physics of Solids 58 (2010) 1628-1645.

[12] A. Ridruejo, C. González, J. Llorca, Damage localization and failure locus under biaxial loading in glass-fiber nonwoven felts, International Journal for Multiscale Computational Engineering (2012), in press.

[13] A. Ridruejo, Mechanical behavior of nonwoven felts, PhD thesis, Polytechnic University of Madrid, 2011.

[14] A. Ridruejo, C. González, J. Llorca, A constitutive model for the mechanical behavior of nonwoven fabrics, International Journal of Solids and Structures (2011), submitted for publication.

[15] A. Ridruejo, C. González, J. Llorca, Micromechanisms of deformation and fracture of polypropylene nonwoven fabrics, International journal of Solids and Structures 48 (2011) 153-162.

[16] D.R. Petterson, On the mechanics of nonwoven fabrics, PhD thesis, MIT, 1959.

[17] J. Planas, G.V. Guinea, M. Elices, Constitutive model for fiber-reinforced materials with deformable matrices, Physical Review E 76 (2007) $041903-$ 041909.

[18] H.L. Cox, The elasticity and strength of paper and other fibrous materials, British Journal of Applied Physics 3 (1952) 72-79.

[19] J.C. Simó, T.J.R. Hughes, Computational Inelasticity, Springer-Verlag, 1998.

[20] J. Lemaitre, A Course on Damage Mechanics, Springer-Verlag, 1992.

[21] Abaqus, Users' Manual, version 6.7, Abaqus, Inc., 2008.

[22] Z.P. Bažant, B.H. Oh, Crack band theory for fracture of concrete, Materials and Structures 16 (1983) 155-177.

[23] Z.P. Bažant, J. Planas, Fracture and Size Effect in Concrete and Other Quasibrittle Materials, CRC Press LLC, 1998.

[24] M.A. Crisfield, Non-Linear Finite Elements Analysis of Solids and Structures, John Wiley \& Sons, Ltd., 1991.

[25] E. Totry, C. González, J. Llorca, J.M. Molina Aldareguía, Mechanisms of shear deformation in fiber-reinforced polymers: Experiments and simulations, International Journal of Fracture 158 (2009) 197-209. 